\title{
Belphégor
}

Littérature populaire et culture médiatique

$18-2$ | 2020

Roman Populaire Espagnol - Roman historique, 1900-1950

\section{La Saga de Los Aznar}

Una serie mítica en la Ciencia Ficción española

\section{Carlos Quintana Francia}

\section{(2) OpenEdition}

\section{Journals}

\section{Edición electrónica}

URL: http://journals.openedition.org/belphegor/3136

DOI: 10.4000/belphegor.3136

ISSN: 1499-7185

\section{Editor}

LPCM

\section{Referencia electrónica}

Carlos Quintana Francia, «La Saga de Los Aznar », Belphégor [En ligne], 18-2 | 2020, mis en ligne le 14 décembre 2020, consulté le 28 avril 2021. URL : http://journals.openedition.org/belphegor/3136 ; DOI : https://doi.org/10.4000/belphegor.3136

\section{Este documento fue generado automáticamente el 28 abril 2021.}

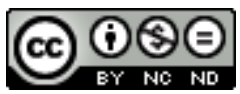

Belphégor est mis à disposition selon les termes de la Licence Creative Commons Attribution - Pas d'Utilisation Commerciale - Pas de Modification 4.0 International. 


\title{
La Saga de Los Aznar
}

\author{
Una serie mítica en la Ciencia Ficción española
}

\author{
Carlos Quintana Francia
}

1 Cuando me dijeron de escribir este artículo, me entraron serias dudas de cómo enfocarlo. Lo cierto es que ya he publicado varios ensayos sobre este mismo tema, con enfoques muy diferentes y la verdad es que no sabía cuál emplear en el caso presente.

2 A veces me he centrado en explicar cómo me introduje en las novelas de Ciencia Ficción, en aquellos tiempos en que aún no se había decidido cómo llamarlas. En otras ocasiones he tratado de situar esta serie en el entorno español de los años 50 y justificar por qué en 1978 le concedieron en la Eurocon de Bruselas el premio a la Mejor Serie Europea de Ciencia Ficción.

3 También he dado, en el marco de la Hispacones, algunas charlas sobre la vida y obra de su autor, George H. White, seudónimo tras el que se ocultaba el valenciano Don Pascual Enguídanos Usach, tema éste que trataremos más adelante.

4 Y evidentemente un aspecto que ha salido siempre ha sido el contenido de la Saga de los Aznar, tanto en su aspecto puramente literario como sobre todo en el argumental, hablando no solo de los personajes sino sobre todo del desarrollo de su trama a través de las sucesivas novelas y de los descubrimientos y avances tanto tecnológicos como sociales e incluso metafísicos.

5 Pero después de haber releído los párrafos anteriores, creo que lo mejor será mezclar todos los puntos mencionados, empezando por el principio, es decir, al comienzo de los años 50.

6 En aquellos tiempos no existía en España ninguna literatura de Ciencia ficción, ni tan siquiera existía ese nombre. Dejando aparte algunos autores actualmente poco conocidos, tales como José de Elola y Jesús de Aragón, este género era totalmente desconocido, a no ser que queramos incluir en él colecciones como Doc Savage, aunque fuesen más de aventuras que de otra cosa.

7 Y fue en 1953/54 cuando apareció la colección "Futuro", obra personal del popular autor de El Coyote: José Mallorquí y casi simultáneamente Editorial Valenciana lanzó al mercado "Luchadores del Espacio", que en un principio fue también una apuesta 
personal de otro escritor de las llamadas "novelas de a duro", conocidas bastantes años después como "bolsilibros".

8 Se trataba de un novelista que llevaba tiempo colaborando en la colección "Comandos", de esa misma editorial, bajo el seudónimo de George H. White, si bien su verdadero nombre era Pascual Enguídanos Usach, natural de Lliria, Valencia.

9 Podríamos decir que sus dos primeras novelas: Los hombres de Venus y El planeta misterioso están muy influidas por el espíritu de "Comandos", pero poco a poco esto va evolucionando y a partir sobre todo de la cuarta, "Cerebros electrónicos", entramos ya en la Ciencia Ficción, en concreto en el apartado que posteriormente se conocería como "Space-ópera".

10 He dicho anteriormente que esta nomenclatura no era popular en nuestro país, por lo que mis amigos y yo las llamábamos Las aventuras del Miguel Angel y es precisamente aquí donde aparece una de las características que harían a esta serie distinta de todas las demás. Su principal protagonista, Miguel Angel Aznar de Soto, era español, caso muy raro entre todos los personajes de las novelas de este tipo, que eran anglosajones. Y en las sucesivas novelas seguirían predominando cada vez más los apellidos hispanos: Valera, Ferrer, Castillo, Contreras, Anglada, etc., coexistiendo evidentemente con los Balmer, Steffansson, Tierney, Mac Lane y otros.

11 Como anécdota personal, me gustaría comentar que, en mi perspicaz mentalidad juvenil, ya adiviné que aquel autor no podía ser americano, sino que eso de George $\mathrm{H}$. White debía ser una traducción de Jorge Hernández Blanco o algo así, con lo que no iba desencaminado, si bien no acerté ni el nombre ni ninguno de los apellidos.

Antes de continuar adelante y entrar ya en detalles concretos, debo hacer una precisión que es que la Saga volvió a ser reeditada en los años 70 y para ello, sobre todo las primeras novelas fueron casi totalmente reescritas por su autor, por lo que algunos comentarios pertenecerán indistintamente a una u otra de esas dos ediciones. Más adelante hablaremos de que a partir del año 1999 se produjo una tercera edición, a cargo de Editorial Silente, pero como digo, de esto hablaremos más adelante.

Situémonos pues, a principios de los años 50 y permítaseme recordar la primera portada de "Luchadores del Espacio" con la que me encontré casualmente en mi kiosco, que era ya el número tres de la colección. Aparecía un extraño robot tal como puede verse en la ilustración 1. 
Fig. 1: Portada de una de las primeras novelas de la Saga

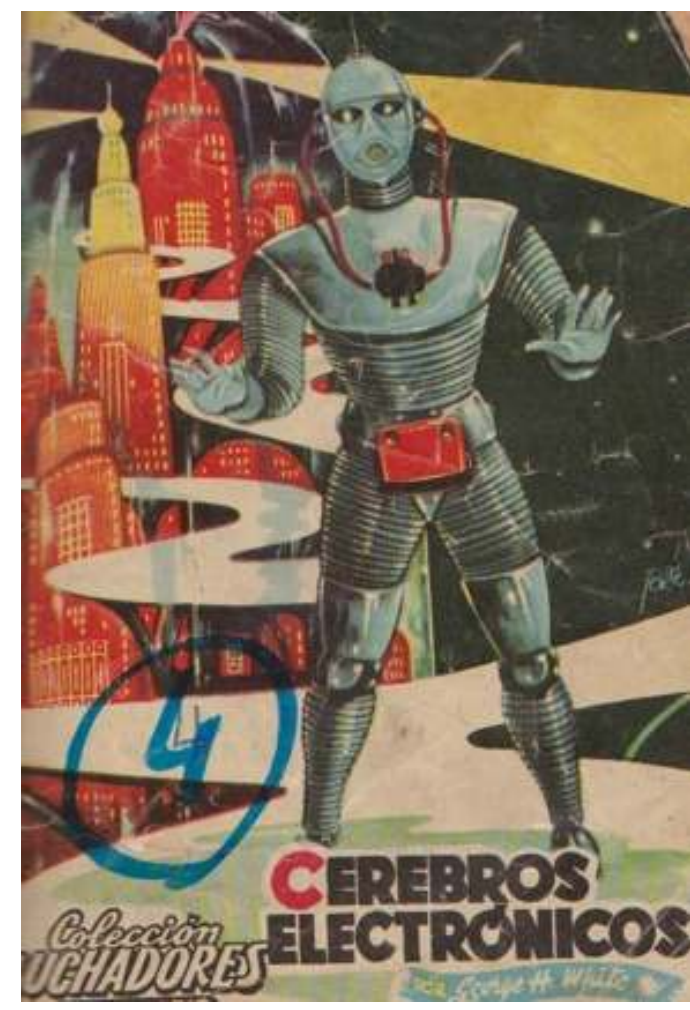

14 Al igual que en el resto de aquellas primeras novelas, la fantasía corría a raudales, juntamente con una falta de rigor científico más que notable. Vaya como ejemplo que la mayor parte de la acción de esta novela ocurría en un planeta errante que atravesaba el Sistema Solar ¡a mayor velocidad que la de la luz!

15 Pero esto no era obstáculo para que "el sentido de la maravilla" impregnara todas sus páginas. En la segunda edición de los años setenta, el autor modificó gran parte de estas primeras novelas y la base científica era ya mucho más aceptable, aunque, por ejemplo, Venus seguía apareciendo como un mundo en pleno período jurásico, con selvas gigantescas, dinosaurios, reptiles voladores, etc., cosa ciertamente muy en boga en este tipo de literatura, como podemos ver en el ciclo de Marte de Edgar Rice Burroughs, el autor de Tarzán, o en los cómics de Flash Gordon.

16 Es muy posible que la idea de la primera edición fuese que todas las novelas estuviesen escritas por George $\mathrm{H}$. White, pero pronto se demostró que el autor no podía hacer frente a una novela semanal, por lo que fueron introduciéndose otros autores, como Alf Regaldie, pero, así y todo, de las 234 novelas que se publicaron, 46 estaban firmadas por George H. White, si bien sólo 30 eran propiamente de la Saga de los Aznar. Como detalle accesorio, podemos explicar que en los últimos tiempos de "Luchadores del espacio" aparecieron otras 22 novelas del mismo autor, aunque firmadas con el pseudónimo de Van S. Smith.

Posteriormente, en la segunda edición de los años setenta, la historia se prolongó hasta un total de 59 títulos, aparte de dos que no llegaron a publicarse por el cierre de la editorial, de los que desgraciadamente sólo se conservan los títulos: El gran miedo y Escuadrón Delta. 
18 Hechas todas estas consideraciones de tipo general, vamos a entrar en detalles de la obra en si.

19 Empecemos por decir que el argumento se desarrolla a partir de medianos del siglo XX hasta muchos miles de años en el futuro, ya que los fenómenos de la relatividad hacen que el tiempo transcurra diferente para los protagonistas que viajan a grandes velocidades por el espacio, frente al resto de la Humanidad que permanece en la Tierra o en otros planetas.

20 Y ahí aparece una de las características de la serie: que sus personajes nacen, viven y mueren y son sus descendientes los que van viviendo las sucesivas aventuras, que nos van siendo relatadas lineal y cronológicamente.

21 Tal como hemos dicho más arriba, el primer protagonista en Miguel Angel Aznar de Soto, piloto americano de origen español, que a las siete u ocho novelas se ve sustituido por su hijo Fidel Aznar Contreras en tres o cuatro novelas, y así sucesivamente.

22 Pero no se crea que los protagonistas van siendo los descendientes directos de aquel primer Aznar, sino que van apareciendo otros varios que pueden ser militares, científicos, políticos, periodistas, simplemente ciudadanos "de a pie" o incluso un futbolista.

Las primeras novelas transcurren básicamente en nuestro Sistema Solar, con un enemigo común que continuará existiendo a lo largo de toda la serie, Se trata de los thorbods, conocidos también como "hombres grises", por el color de su piel. En la ilustración 2 pueden verse diversas interpretaciones gráficas de estos seres, dibujadas a lo largo del tiempo por José Luis Macías, el primer portadista de la Saga, y posteriormente por diversos aficionados a la misma.

Fig. 2: Diversas versiones gráficas de los Thorbods u Hombres grises

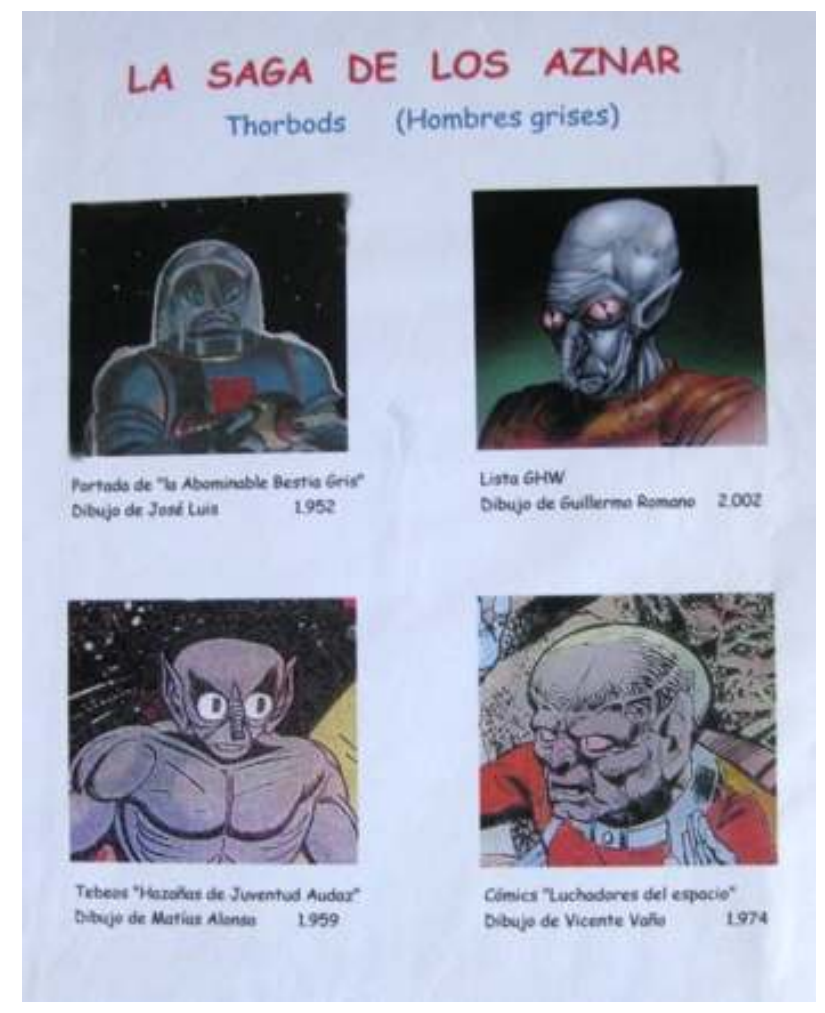


24 Aprovechemos para hacer un inciso y comentar que aparecieron también tres colecciones de cómics, algunos de cuyos ejemplares pueden verse en la ilustración 3.

Fig. 3: Las tres colecciones de cómics
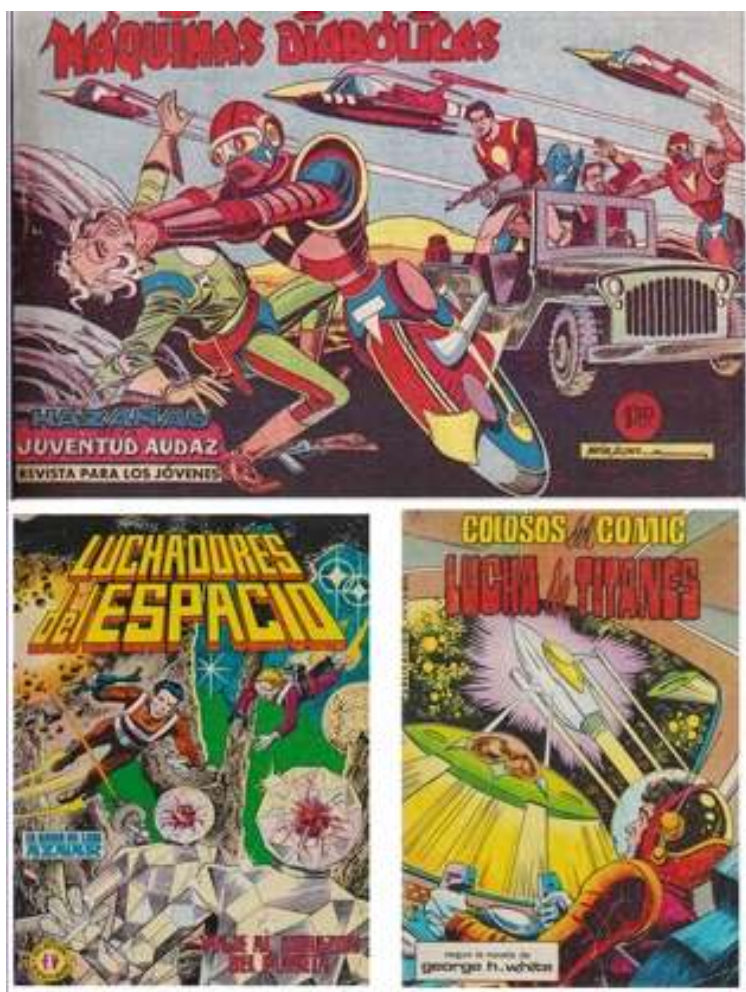

Volviendo a la historia de la Saga el caso es que después de diversas guerras y confrontaciones, la Humanidad es derrotada por los Thorbods y tan solo unas seis mil personas pueden huir de la Tierra a bordo del Rayo (Figura 4), una fabulosa nave espacial que Miguel Angel Aznar y sus amigos han traído de Ragol, aquel planetoide errante que hemos mencionado en páginas anteriores. 


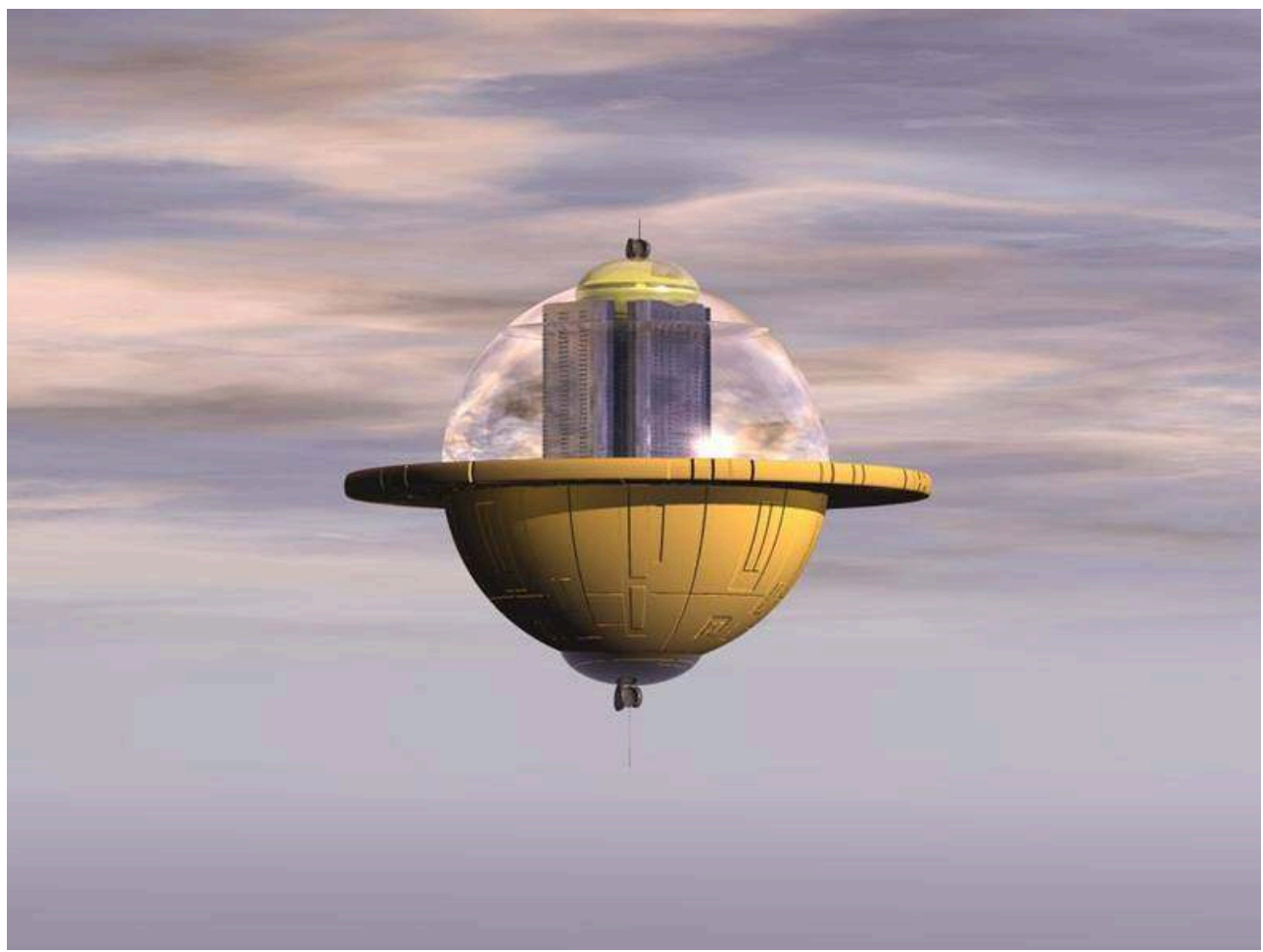

Hasta ese momento, sobre el año 2.600, el desarrollo bélico estaba basado en naves espaciales tipo platillos volantes o cohetes interplanetarios, dotados de motores atómicos y cuya principal arma eran los rayos $\mathrm{Z}$, especie de rayos $\mathrm{X}$ capaces de desintegrar los metales.

Pero Miguel Angel y sus amigos se habían traído de Ragol un nuevo metal: la dedona, de densidad miles de veces superior al hierro y resistente a los rayos Z. Pero su escaso número no les sirven para hacer frente a los thorbods y al final de la La abominable bestia gris, número 8 de la colección, aquellos seis mil terrestres, que por cierto son madrileños en su mayoría, se ven obligados a huir en busca de un mundo nuevo fuera del sistema Solar, dejando a los restos de la Humanidad esclava de los thorbods.

Como un nuevo Moisés, Miguel Angel conduce al Rayo cuarenta años a través del espacio hasta llegar a un gigantesco mundo apto para la raza humana. Aquí el protagonismo empieza a pasar a su hijo Fidel que termina casándose con una indígena de aquel nuevo mundo que bautizan con el nombre de Redención.

Ocurren nuevas aventuras motivadas porque aquel gigantesco planeta es hueco y en su interior habita una raza basada en el silicio, los hombres de cristal de la ilustración 5, a la que finalmente consiguen derrotar. 
Fig. 5: Diversas versiones gráficas de los Hombres de silicio

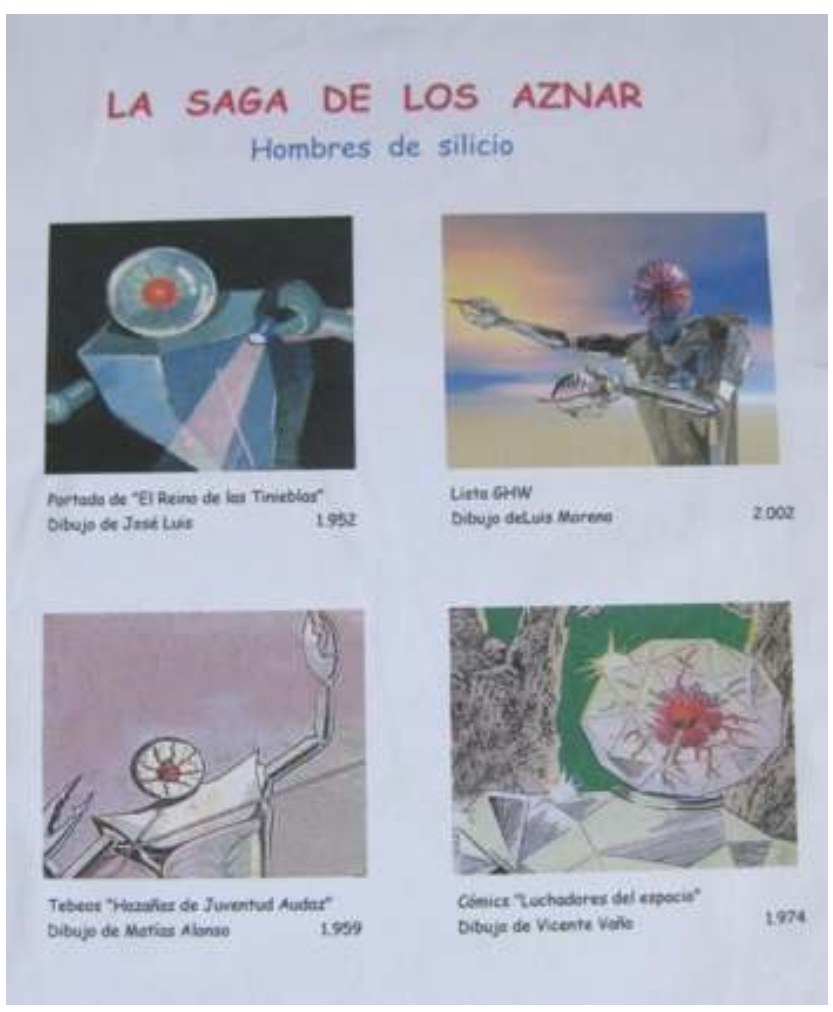

Los españoles mezclan su sangre con los indígenas de Redención en una serie de episodios que recuerdan a Conquista de América por los españoles, creando así un nuevo pueblo dispuesto a regresar a la Tierra y liberar a sus hermanos de la esclavitud a la que sin duda seguían sometidos.

31 Aclaremos que estos episodios fueron escritos a medianos de los años 50 y que la exaltación de los valores patrios estaba muy bien vista por el Régimen, por no mencionar los problemas que, en otro caso, pudieran plantearse con la Censura.

El caso es que en aquel sistema solar todos los planetas son huecos y descubren uno del tamaño aproximado de la Luna que es enteramente de dedona, metal que no sólo es resistente a todas las armas conocidas, sino que además al ser inducido por unas corrientes eléctricas especiales repele la atracción de los planetas.

Este planetillo es bautizado con el nombre de su descubridor, el astrónomo Valera, y convertido en un gigantesco autoplaneta será la base de la Armada Sideral que a lo largo del resto de la serie llevará sus habitantes por todos los confines de la galaxia.

No creemos que sea aquí el lugar ni el momento de ir detallando los diversos episodios de la serie, digamos tan solo que Valera regresa a la Tierra y consigue derrotar a los hombres grises e iniciar la liberación de la humanidad, gracias sobre todo a la mejor calidad de la dedona y de los rayos $\mathrm{Z}$ de los valeranos.

Pero el autor se saca aquí un nuevo as de la manga y aparece un nuevo pueblo, los nahumitas, enemigo ancestral de los thorbods que han "venido a destruir el Mundo", como se titula el número 16 de la colección.

La Armada Imperial de Nahúm viene provista de corazas del mismo tipo de dedona que la de los valeranos, por lo que ha de aparecer una nueva arma: los torpedos autómatas, fabricados también de dedona e inmunes por lo tanto a los rayos $\mathrm{Z}$. Se suceden 
tremendas batallas siderales, en las que intervienen centenares de miles de buques que lanzan y esquivan millones de torpedos. Esta será una de las características de la Saga; los navíos y sus proyectiles se miden en centenares de miles o millones...

Finalmente, los nahumitas son derrotados, pero la Tierra, Marte y Venus quedan impregnados de radioactividad y se impone un éxodo en masa des terrestres, rumbo a Redención.

Podemos aprovechar para mencionar aquí que los protagonistas de las tres novelas de este ciclo no son los descendientes directos de los Aznar, sino respectivamente una capitana del Servicio de Inteligencia de la Armada, un comandante de dicha Armada y un coronel del Ejército Autómata, junto con esclavos o exesclavos recién liberados.

Esta tónica continuará en el siguiente ciclo, cuando al regreso a Redención se encuentran con que los hombres de cristal se han resucitado y dominan la totalidad del planeta.

Dura otras tres novelas el triunfo sobre estos enemigos y aquí los protagonistas siguen siendo personas "normales": un teniente de las Fuerzas Especiales, unos naturalistas y un ingeniero y su secretaria.

41 Tal como hemos insinuado anteriormente, una de las características de La Saga de los Aznar es que la tecnología y la sociedad va avanzando paulatinamente, justificando o al menos intentándolo, todos y cada uno de los diversos avances que se van produciendo.

Todo ha ocurrido tan solo en dos sistemas solares, el nuestro y el de Redención y como razas extraterrestres han aparecido tan solo los thorbods y los nahumitas, ambos de carbono, y los silíceos, detalle en línea con las teorías pseudocientíficas de la época, en que se hablaba de biologías basadas en el silicio o el titanio, elementos ambos tetravalentes al igual que el carbono.

43 Uno de los detalles más importantes de la Saga es el modo de vida de la sociedad. Los adelantos científicos han liberado al hombre del castigo bíblico de "ganarse el pan con el sudor de su frente". Es el Estado quien provee de todo lo necesario a cambio tan solo del SOT (Servicio Obligatorio del Trabajo) de duración variable según la época y las circunstancias. Pero no toda la Humanidad se conforma con no hacer nada y así tenemos que nunca las artes ni las ciencias han estado tan desarrolladas, ya que no faltan personas que sea por curiosidad intelectual, o por ansias de poder o por vocación o por querer sobresalir de la masa y obtener ventajas adicionales, se dedican a las más diversas profesiones y contribuyen así al avance de la Humanidad.

Cuando hay un problema general, como por ejemplo una guerra o una crisis de otro tipo, todo el mundo tiene que arrimar el hombro y sacrificarse por el bien común.

45 Si siguiéramos ahora el orden cronológico, nos tocaría hablar de las cuatro novelas del ciclo de Nahum, pero vamos a pasar rápidamente por él, comentando simplemente algunos detalles.

46 Ahora el protagonismo sí que recae básicamente en un miembro de la familia Aznar, que de simple cadete llega a ser finalmente Almirante Mayor de Valera, tras una serie de aventuras que a ratos recuerdan muy mucho las de Flash Gordon, con sus princesas y reinas, sus circos con espectáculos sangrientos, etc. Pero enseguida volvemos a los clásicos argumentos de George $\mathrm{H}$. White, con las luchas contra el Imperio de Nahum, cuyos emperadores practican el cambio de cerebros de un cuerpo viejo a uno joven, 
práctica que aparece repetidas veces en la serie, siendo siempre repudiada como moralmente condenable.

En este ciclo aparece también una de las características típicas de la Saga: la mala estrella de las mujeres de los Aznar y de la familia en general, ninguno de los cuales muere en la cama. Aquí todos los miembros importantes de la familia, que ocupan la jefatura de Valera, son linchados por el populacho que los considera culpables de la rendición del autoplaneta a los nahumitas. Posteriormente la novia de Miguel Angel, el benjamín de la familia, muere en las arenas del circo, etc. Triste sino el de los Aznares, que en varias ocasiones adquiere aires de auténtica tragedia griega...

De los planetas nahumitas la Saga se traslada a los planetas que fueron cuna de los thorbods, para vivir allí nuevas aventuras con la aparición de hombres-planta, seres de rudimentaria inteligencia totalmente dominados por los hombres grises.

Lo más destacable de este ciclo es la aparición de la técnica de miniaturización de la materia, que hace posible comprimir los navíos y torpedos al tamaño de un juguete, lo que permiten a los valeranos derrotar nuevamente al renacido Imperio de Nahúm.

Evidentemente esta técnica presenta una serie de ventajas prácticas en todos los ámbitos de la industria, pero no vamos a comentarlas aquí. Digamos solamente que, como siempre, el autor intenta explicar los fenómenos en que se basa esta técnica, siendo ésta una las características que ya hemos apuntado anteriormente en el sentido de que todos los avances técnicos que se van presentando intentan ser explicados, con mayor o menor fortuna, pero siempre con una base lógica y razonada, al contrario de otras novelas de Ciencia Ficción donde las técnicas y "los inventos" aparecen sin ningún tipo de explicación ni justificación.

51 Volviendo a los argumentos de la serie, vamos a pasar rápidamente por encima de unas cuantas novelas para no hacernos demasiado pesados. Corresponden al regreso de Valera a la Tierra, con la proclamación de un Imperio Balmer, acaudillado por los miembros de esta familia, descendientes del mejor amigo del primer Miguel Angel.

Derrotado este anacrónico imperio, se regresa a la democracia, pero ante la progresiva degradación del espíritu cívico del pueblo, que busca solo sus comodidades, el Miguel Angel de turno, el que habíamos dicho que llegó de cadete a Almirante Mayor, da un golpe de Estado y dedica todos los esfuerzos a disciplinar la población y crear unas potentes Fuerzas Armadas, para poder estar prevenidos si regresan los thorbods o cualquier otra raza que amenace al Sistema Solar.

Ello provoca un gran descontento en la gente $\mathrm{y}$, cumpliéndose una vez más la mala suerte de las esposas de los Aznar, Carmen, su mujer, muere en un atentado.

Repentinamente aparece la Bestia Gris e invade la Tierra, pero finalmente son derrotados.

Llegados a este punto debemos hacer mención a que en las novelas de George H. White existe siempre una confrontación más o menos declarada entre las masas y las élites que "se sacrifican", dedicando su vida y sus esfuerzos al desarrollo general. En línea con todo esto, la figura de los políticos no suele salir bien parada, ya que en muchos casos están más pendientes de seguir en sus puestos que del bien común, efectuando concesiones para obtener mayor número de votos, lo cual al final conduce a una indolencia y una falta de profesionalidad muy perjudicial para la nación. 

correspondería con la novela 33 de las aventuras de los Aznar. En este episodio el autoplaneta Valera regresa a Redención, para encontrarse con los "eternos" una especie de ciborgs de acero en cuyo interior vive un celebro humano, habiendo obtenido así una falsa inmortalidad (Figura 6). 
Fig. 6: Los Eternos, ciborgs con un cerebro humano

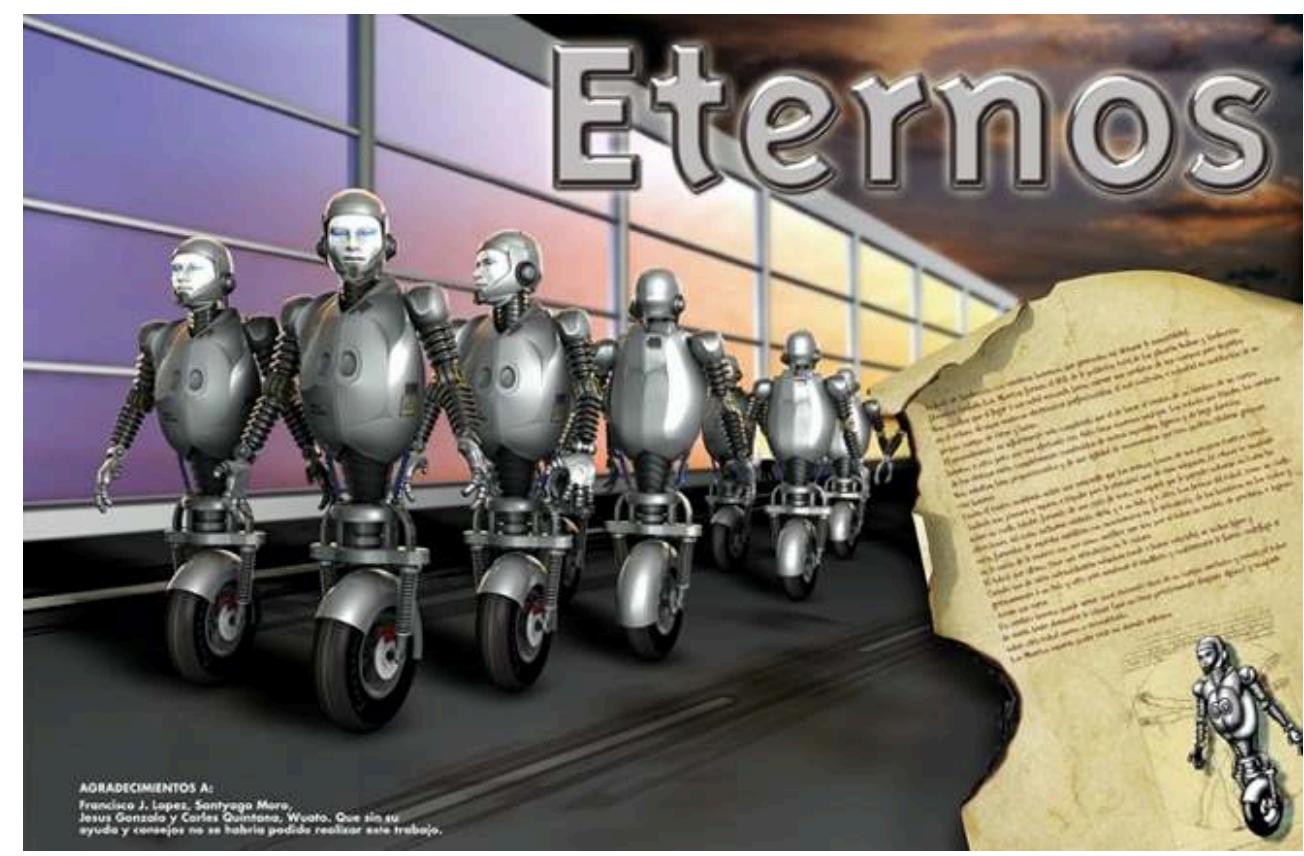

Tras una serie de confrontaciones, Valera se declara independiente y abandona el sistema redentor..." Rumbo a lo desconocido", como ordena el Almirante Aznar en la última página de la novela.

Y aquí terminó la colección, posiblemente porque su autor, George H. White, debía estar ya cansado de esta serie y además acababa de firmar por otra editorial.

Tuvieron que transcurrir casi veinte años para que, en 1974, Editorial Valenciana volviera a editar estas novelas, que acabaron titulándose La Saga de los Aznar y que hasta 1978 fueron reeditándose primero y continuando posteriormente hasta llegar a 59 títulos, e interrumpiéndose súbitamente por el cierre de la empresa. Tan repentino fue ese cierre que quedaron pendientes de publicar dos novelas de las cuales sólo se conservan los títulos: El gran miedo y Escuadrón Delta, ya que los originales desaparecieron en las profundidades de los almacenes...

Pero durante estos veinte años se habían producido muchos cambios, tanto en la mentalidad del autor como en el modo de vida del país.

El autor, con mucha más experiencia que cuando empezó a escribir novelas de cienciaficción, decidió reescribir las cuatro primeras, resumiéndolas en tres y con un mayor rigor científico. También modificó detalles de otras novelas, pero de un modo menos importante.

72 El resultado no ha quedado claro, ya que los aficionados a la Saga estamos divididos entre los que leímos la primera edición y los que entraron directamente en la segunda. En general todos estamos de acuerdo en que técnicamente es mejor la segunda, pero ha perdido la frescura y el "sentido de la maravilla" de aquellas inocentes novelitas de principios de los cincuenta.

73 Como también la situación política había cambiado mucho, desapareció el sentido epopéyico en que repetidas veces se comparaban episodios de la Saga con análogas situaciones de la Conquista de América por lo españoles y otras referencias a los más heroicos episodios de la Historia de España. 
Lógicamente los castos besos del final de la novela, habían cambiado por escenas de cama, aunque tan sólo esbozadas, sin entrar nunca en detalles...

Vayamos, pues, a la segunda parte de la Saga, si bien dando un giro al modo de narrarla.

Hasta ahora hemos ido comentando los argumentos, aunque fuera de modo superficial y resumido, intercalando sobre la marcha diversos detalles que creemos importantes, tanto tecnológicos como sociales. Pero a partir de ahora hablaremos tan solo de los conceptos generales, ya que en ellos se incorporan una serie de temas y novedades muy interesantes.

Desde un punto de vista astronómico, digamos en el autor se saca de la manga dos cosas: un circumplaneta y un hiperplaneta.

El primero, el circumplaneta, nos puede recordar el Mundo Anillo de Larry Niven, puesto que se trata de un colosal anillo toroidal de materia sólida, con un sol en su centro y con un diámetro algo superior a la órbita de la Tierra, creado artificialmente por los bartpuranos, especie humana muy avanzada técnica y filosóficamente. En este gigantesco mundo transcurrirán un buen número de los episodios de esta segunda parte de la serie.

Estos bartpuranos dan un giro al aspecto religioso de la Saga. No profesan una verdadera religión sino un principio filosófico muy elevado, hasta el punto de que su respeto por todo tipo de vida ha hecho que ocurra lo siguiente. Siglos después de haber construido el Cincumplaneta y al verse amenazados por las mantis, una raza de insectos sociales muy evolucionada, parecidos a las hormigas, o mejor dicho a nuestras "mantis religiosas", de ahí su nombre, no quieren luchar contra ellas, a pesar de que su tecnología bélica es muy superior y deciden "ausentarse".

¿Qué quiere decir "ausentarse" ...?

He aquí un concepto nuevo que merece una amplia explicación.

Este nuevo concepto es ¡la máquina karendón!

La karendón es una máquina que es capaz de leer la composición atómica de un cuerpo, almacenar su fórmula y seguidamente desintegrar ese cuerpo. Y lo importante es que posteriormente basta con leer la cinta en que se han almacenado esos datos, para que la karendón repita el proceso a la inversa e integre de nuevo el cuerpo original.

Este proceso es válido no sólo para los cuerpos inorgánicos, sino también para los seres vivos. El alma, la vida o como queramos llamarle, vuelve al ser recién integrado y éste sale vivito y coleando de la máquina.

5 Pero aquí el autor introduce una premisa muy importante, Un ser vivo previamente desintegrado puede ser recuperado de nuevo tantas veces como se desee, pero únicamente la primera copia saldrá vivo. Alma sólo hay una y una vez se ha "reintegrado" en un cuerpo ya no puede ir a otra copia del mismo y por lo tanto todos esos nuevos cuerpos aparecerán sin vida, muertos.

Esta circunstancia viene a demostrar la existencia del alma y la filosofía bartpur cree en la reencarnación. Téngase en cuenta que por aquellos años setenta estaban de moda las teorías orientales, budistas, etc. y es de suponer que el autor hubiese sido influido por ellas.

Como decíamos, los bartpures han recurrido a la karendon para ausentarse y dejar en paz a los insectos en espera de que otro pueblo llegue al Circumplaneta para solucionarles el problema. 
88 En este momento ha surgido uno de los temas más debatidos por los aficionados a la Saga. ¿Si los bartpures eran tan "buenos" que sus principios no les permitían matar a las mantis, porqué esperaron que llegara una raza más "primitiva", los valeranos en este caso, que no tuviera tantos escrúpulos y luchara a muerte contra los insectos...?

Dejando aparte estas disquisiciones, digamos que las máquinas karendón tienen infinidad de aplicaciones, desde las modestas "despenseras" que pueden prepararte un suculento desayuno, hasta las "karendon grada", de centenares de metros de longitud, que son capaces de integrar, uno tras otro, toda una flota de destructores siderales, a cambio, claro está, de un elevadísimo consumo de energía.

Otra versión que aparece posteriormente es la "karendón traslator", que una vez desintegrada una persona, envía sus datos por radio y puede ser reintegrada en otro lugar, sin importar la distancia. Esta versión deja pequeña a los transportadores de las de Star Trek.

91 También puede emplearse para "curar" a los heridos, a base de desintegrarlos y volverlos a integrar en una versión anterior al accidente.

92 Pero en este caso ocurre otro fenómeno. Se supone que los recuerdos de la persona están almacenados en su cerebro, no en su alma. $Y$ consecuentemente, el ser reintegrado en una versión anterior saldrá con los recuerdo del momento en que fue desmaterializado, que puede ser días, meses o años antes y no con los actuales.

$93 \mathrm{Y}$ ahí entra en escena otro dispositivo existente ya antes de conocerse las karendón: la Máquina Psi.

94 Estas máquinas Psi fueron creadas en su momento para facilitar la instrucción y el aprendizaje de las personas. Habiéndose estudiado ya muy a fondo la estructura del cerebro, la Ciencia había sido capaz de detectar exactamente para que servía cada uno de sus componentes y actuando sobre determinadas zonas podía enseñarse al sujeto un idioma nuevo, la tabla de multiplicar o lo que se quisiera, incluyendo experiencias de otras personas.

95 Se podía así formar excelentes médicos, astronautas o futbolistas, pero hay que tener en cuenta que, aunque la persona tuviera todos los conocimientos de sus maestros, en el fondo eran sus condicionantes tanto físicos como mentales y sobre todo su carácter y sus ganas de trabajar y superarse, los que determinaban su trayectoria futura y su éxito en la vida.

96 Una versión malévola de estas máquinas sería inducir en los ciudadanos un sometimiento total a la Autoridad, un odio o una admiración hacia otras personas o pueblos, etc. Digamos que esto también se presenta en alguna de las novelas de esta segunda parte, si bien no vamos a entrar en detalles.

97 Pero volviendo a las karendón, si se combinan sus posibilidades con las de la máquina Psi, nos encontramos con que es posible una especie de inmortalidad. Basta con que una persona obtenga una cinta con los datos de su cuerpo a los veinte años, viva tranquilamente su vida y al llegar a una edad en que el cuerpo empiece a fallar, pase por una Psi, que extraiga todos sus recuerdos actuales, se desintegre seguidamente, vuelva a encarnarse con la fórmula de sus veinte años, y sea entonces sometido a una sesión Psi que le actualice su cerebro con todas sus vivencias y recuerdos actuales y ya tenemos a la persona con toda su personalidad actual pero en un cuerpo rejuvenecido. 
98 No obstante, esto no es una inmortalidad, porque el autor se saca de la manga un fenómeno paranormal que hace que llegue un momento en que ya no sea posible hacer nuevas reencarnaciones.

Todo esto que aquí hemos comentado tan rápidamente no ocurre en una sola novela, sino que van apareciendo poco a poco, intentando como siempre dar una explicación lógica, más o menos científica, a cada detalle, lo cual tal como hemos comentado anteriormente, es uno de los elementos característicos de la Saga, cosa poco común en las novelas de este tipo.

Otro aspecto que no podemos soslayar está también relacionado con la filosofía budista o paranormal: Resulta que los bartpures, siendo una raza antiquísima, han desarrolla enormemente sus facultades intelectuales y son, por ejemplo, capaces de leer $\mathrm{y}$ transmitiré el pensamiento. Esto no es problema en su civilización tan avanzada y moralmente elevada como la suya, pero cuando empiezan a mezclarse con los valeranos, los mestizos descendientes están también dotados de facultades semejantes, aunque menos potentes, lo cual hace muy difícil su coexistencia con los humanos “normales" y son, incluso, objeto de discriminación.

La moral de estos mestizos es mucho más relajada que la de los valeranos y por ejemplo no tiene problemas con establecer relaciones amorosas o sexuales con otra persona con la que telepáticamente descubran una atracción mutua. Y como no le dan demasiada importancia a las infidelidades, éstas son frecuentes y normales, pero esto no es aceptado por los rígidos valeranos. Como ejemplo podríamos citar el caso de uno de los superalamirantes, Miguel Ángel Aznar Bogani, que casado con una mujer de estas características, acaba divorciándose, mientras que ella se une con un sobrino del almirante.

Aparecen también otros conceptos que hoy en día son muy frecuentes en las narraciones de CF, pero que no lo eran tanto allá por los años 70. Se trata, por ejemplo, de la antimateria, de las ondas gravitacionales, de viajar por el hiperespacio o por el subespacio, etc.

103 Efectuando pruebas de como desplazarse por el hiperespacio, se producen algunos fallos y nos encontramos con un par de viajes en el tiempo, uno a la época del Diluvio Universal y otro a los finales de la Segunda Guerra Mundial (Figura 7). 
Fig. 7: Un viaje en el tiempo (portada de la novela $n^{\circ} 16$ de la Ed. Silente)

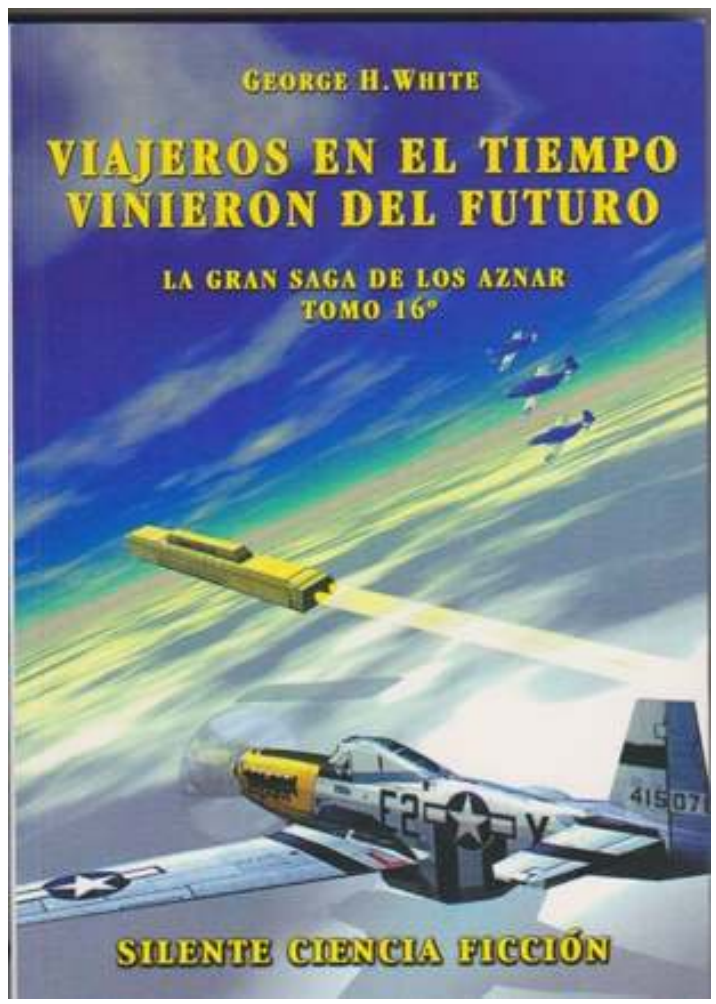

No hay que decir que suponemos que para George H. White debió ser un "divertimento" regresar a sus primeros relatos bélicos en la colección "Comandos", si bien en este caso es un episodio más bien trágico, ya que ocurre en el bombardeo de Dresde.

Así sigue desarrollándose la Saga hasta llegar a las últimas novelas, en las que aparece el Hiperplaneta, una colosal esfera de Dysson en cuyo interior cabe todo un sistema solar.

Desgraciadamente en este momento se produjo el cierre repentino de la Editorial Valenciana, y tal como ya hemos comentado al principio, la serie quedó interrumpida en el número 59: El refugio de los dioses, en cuya contraportada se llegó a anunciar el siguiente ejemplar $\mathrm{El}$ gran miedo, el cual no llegó a editarse y además se perdieron los originales, habiendo sido imposible recuperarlos. También nos consta, según confesión del propio autor, que había escrito otra novela, Escuadrón Delta, que siguió la misma suerte.

Hablando del Escuadrón Delta, digamos que este en el sobrenombre bajo el cual estamos reunidos un grupo de aficionados a la Saga desde hace ya veinte años. En un principio nos limitábamos a intercambiar mensajes y opiniones sobre todos los aspectos de la Serie, pero después nos dedicamos a escribir relatos y novelas ambientados en ese mismo universo creado tan maravillosamente por Don Pascual Enguídanos, habiéndose llegado a publicar una treintena de volúmenes a cargo de Editorial Silente y de su editor Pedro García Bilbao, gran amigo de todos nosotros. A parte de los publicados, existen más de doscientos relatos más, de extensión muy variable, que están a la disposición de todos aquellos que lo deseen, en https://es.groups.yahoo.com/neo/groups/ghwhite/ info

108 También puede encontrarse una amplia información sobre este tema en www.cienciaficcion.com 
109 En este mismo sentido, no podemos olvidar mencionar las Aznarcones. Se trata de que aprovechando las Hispacones, reuniones anuales que los aficionados a la Ciencia Ficción celebramos cada año en alguna ciudad española, incluimos en ellas una serie de conferencias y exposiciones sobre La Saga de los Aznar. Como ejemplo adjuntamos en la figura 8 un poster anunciador de la Hispacón de 2002.

Fig. 8: Poster anunciador de la IV Aznarcon

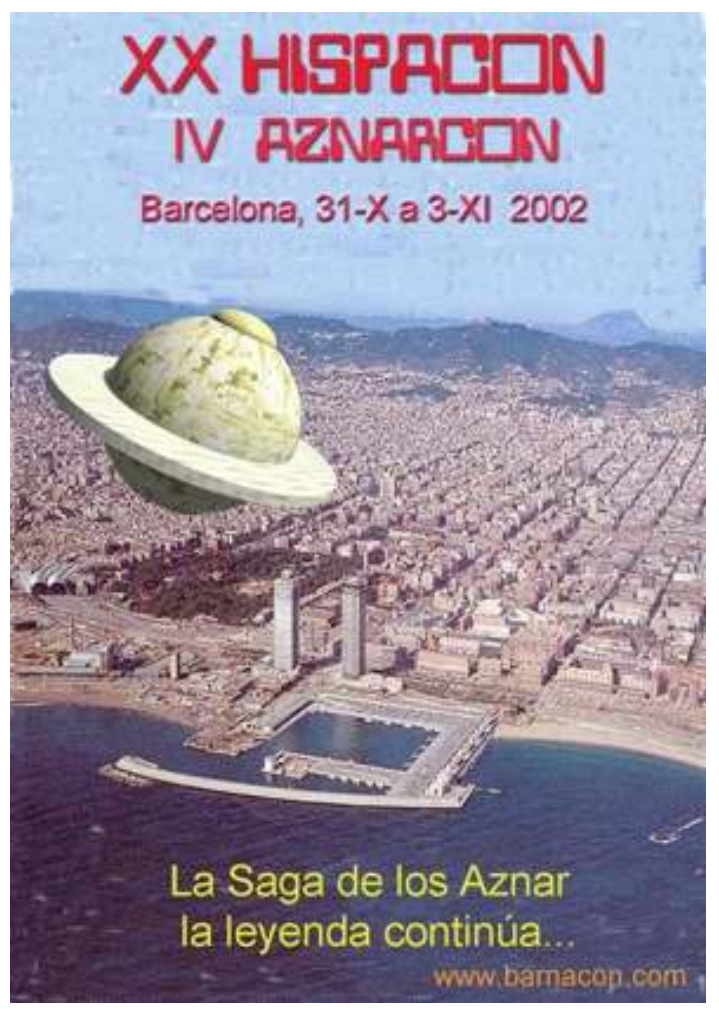

110 Y como complemento a todo lo dicho anteriormente, podemos mencionar que la Saga ha sido llevada al cómic en tres ocasiones, como puede verse en las portadas que adjuntábamos al principio.

111 La extensión reservada a este artículo está llegando a su fin, pero a modo de colofón nos gustaría efectuar un breve resumen de todo lo dicho hasta ahora sobre esta colección mítica dentro de la Ciencia Ficción española :

- Apareció a principios de los años cincuenta, y se han hecho tres ediciones, la última a comienzos del 2000.

- Su autor, Pascual Enguídanos Usach era español, valencianos por más señas, y a causa posiblemente de ello, la mayoría de los personajes son españoles o descendientes de ellos.

- Una de las características de la serie es que, como hemos dicho anteriormente, sus personajes nacen, viven y mueren y la acción va siendo llevada a cabo por sus descendientes más o menos directos o incluso totalmente independientes...

- Aparecen militares, aventureros, científicos, políticos, periodistas, religiosos, incluso futbolistas. No todos son "buenos", también aparecen los malvados de turno, pero no malvados de película, sino seres humanos con sus defectos que les "llevan por el mal camino" 
- Desde un punto de vista literario nos atrevemos a afirmar que su nivel es bastante superior a la mayoría de los escritores de aquella época, tanto desde un punto de vista literario como argumental.

- En este sentido, volvemos a insistir en que todos los adelantos que se van produciendo a lo largo de las cincuenta novelas, tanto tecnológicos como sociales, intentan ser justificados racionalmente, aunque vistos desde la perspectiva actual puede encontrársele multitud de fallos. Pero esto no resta ningún mérito a lo narrado, antes al contrario, nos obliga a contemplarlo a la luz de los conocimientos de la época, tanto generales como el propio autor, que no dejaba de ser un modesto funcionario.

Lo que nos gustaría es haber conseguido que los lectores de este artículo hubieran pasado un rato distraído y que, si se animan a leer alguna de estas novelas, recordarles que Editorial Silente ha publicado la tercera edición, que en este caso va acompañada además de una serie de comentarios sobre diversos puntos interesantes de sus argumentos. En las principales librerías especializadas pueden encontrarse estos ejemplares y no es así, pueden solicitarse directamente a la Editorial.

También puede encontrarse mucha más información en el grupo de correo GHWhite dentro del yahoo o en el Sitio de Ciencia Ficción (http://www.ciencia-ficcion.com). (Literatura/G.H.W.)

Lo que no podemos omitir es un merecido homenaje a George H. White, bajo cuyo seudónimo se encontraba Don Pascual Enguídanos Usach, modesto funcionario valenciano, autor no sólo de esta Saga sino de multitud de novelas de todo tipo, desde bélicas al Oeste, pasando por la novela rosa, etc. Fallecido recientemente, en Marzo de 2006, ha sido objeto de diversos reconocimientos nacionales e internacionales, incluyendo un homenaje popular por parte del Ayuntamiento de Lliria, su pueblo natal (Figura 9).

Fig. 9: Homenaje a Don Pascual en Lliria

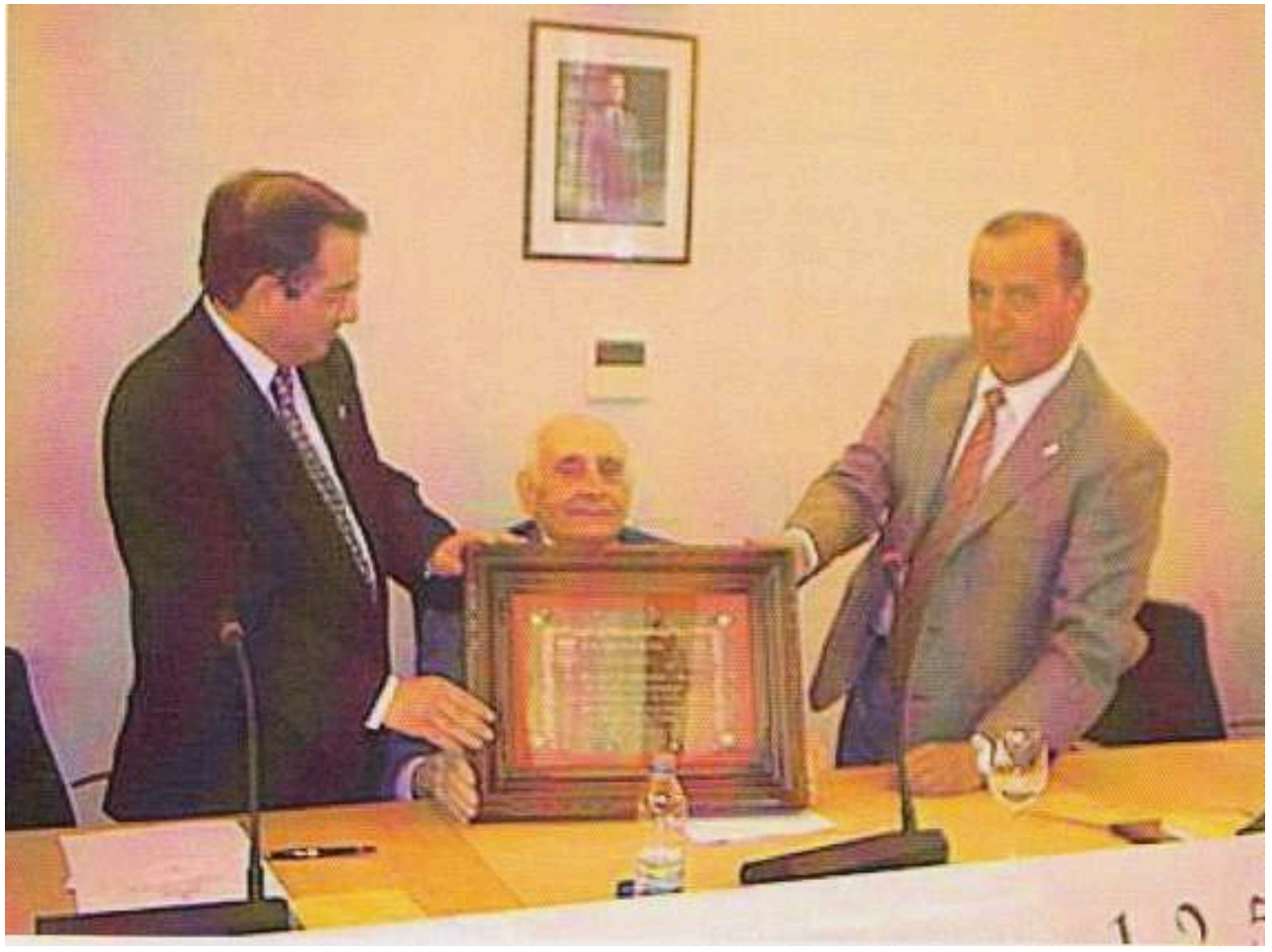


$115 \mathrm{Y}$ ahora sí que hay que poner punto final, no sin agradecer a Belphégor que me haya encargado este artículo, ya que aparte de divulgar una de mis series favoritas, me ha permitido recordar aquellos tiempos en que un muchacho de once añitos adquiría cada viernes las primeras novelas, para comentarlas después con mis amigos y compañeros y mucho después con mi hijo Carles, a quien transmití esta afición y que fue quien un buen día se presentó en casa con un libro titulado Viajes de los Aznar, escrito por Carlos Sáez Cidoncha y Pedro García Bilbao por encargo de Miquel Barceló, y publicado por la UPCF de la Politécnica de Barcelona, el cual puede ser considerado como el detonante de esta tercera explosión popular de La Saga de los Aznar (Figura 10).

Fig. 10: Portada de la primera edición de La Gran Saga de los Aznar

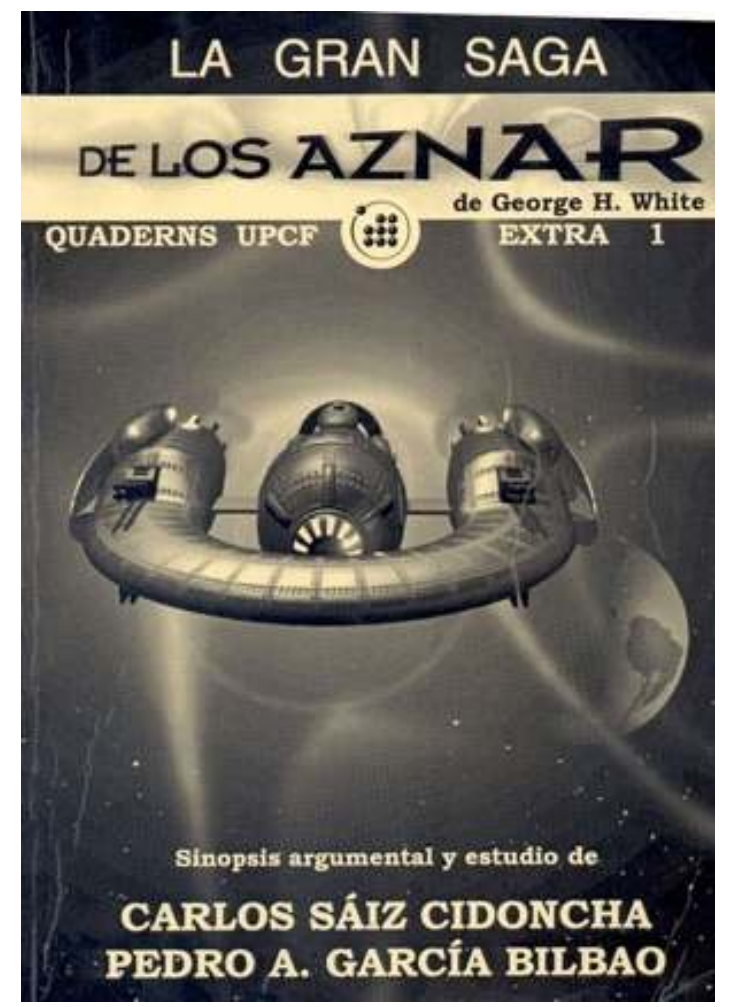

116 A todos ellos también mi agradecimiento.

\section{RESÚMENES}

Se trata de una breve exposición de la serie de novelas de Ciencia Ficción aparecidas en España en los años cincuenta, y que fue reditada y ampliada en los setenta, habiéndosele concedido entonces en la Eurocon de Bruselas de 1978 el premio a la Mejor Serie Europea de Ciencia Ficción. Posteriormente, a principios de los 2000 , tuvo lugar una nueva edición y una gran actividad de los aficionados a través del internet y diversas convenciones

Se trata de unas novelas dentro del estilo que ha venido en llamarse "space opera" y en el artículo se ofrece un resumen de su línea argumental y las características del desarrollo de la 
sociedad humana en el futuro, tanto en el plano tecnológico como social, a lo largo todo ello de varios siglos de historia con las aventuras de Miguel Angel Aznar y sus descendientes.

\section{ÍNDICE}

Palabras claves: colecciones populares, literatura popular, ciencia ficción, La Saga de los Aznar, George H. White

\section{AUTOR}

\section{CARLOS QUINTANA FRANCIA}

Escritor

carlesquin@ono.com 